\title{
Reliability Analysis of Multi-Node SDDC Using Fault Tree
}

\author{
Wan Yaping ${ }^{1,}$, Luo Chenhui $^{1}$ and Liu Zhiming ${ }^{1}$ \\ ${ }^{1}$ School of Computer Science and Technology, University of South China, Hengyang 421001,China \\ aypwan@usc.edu.cn
}

Keywords: Fault Tree, Cloud Computing, Reliability, SDDC

Abstract. The software-defined data center (SDDC), while well understood architecturally, is beginning to reveal some of its benefits beyond agility, speed, and efficiency as organizations deploy and discover other areas of improvement. One critical area which we warmly discussed is reliability. The inaccessible of data and unavailability of computing resources can be frequent, so businesses use many protection techniques to guard against failures. Evaluations of the SDDC reliability have focused mainly on deploying multi-node for the clouding infrastructure which can bear the same works. We use comprehensive failure tree theory to evaluate the reliability and the performance of multi-node cloud computing system. The results show under specific system conditions with maximum functional nodes on the SDDC system, cloud impact on system reliability is nearly larger than the single node of application type.

\section{Introduction}

With the development of the Software Defined Data Center[1,2], more and more researchers focus on reliability and availability [3,4] of cloud computing system. A Software Defined Data Center (SDDC) is an architectural approach to data center design, which leverages a fundamental principle of computer science, abstraction, operating systems, higher-level programming languages, networking protocols. The introduction of an abstraction layer allows systems and services above and below the abstraction layer to operate and innovate independently, while maintaining agreed-upon communication paths and exposing services between layers through well-defined interfaces. An SDDC approach applies the principles of abstraction to deliver an entire data center construct in software, decoupling service delivery from the underlying physical infrastructure.Though the SDDC is blooming, such as VMware, with qualified partners, delivers the EVO:RAIL hyper converged infrastructure appliance via a new business model[5], the price of data loss is even higher. Thus, a reliable cloud computing system is a key to keep computing and data storage service running[6,7]. Enhancing computing, storage, network reliability by adding extra redundancy which sometimes causes design complexities and can be cost-expensive to highly utilized the whole system may lead to enormous benefits. Cloud is a style of computing where massively scalable and flexible IT-related abilities are provided "as services” to external customers using Internet technologies.

Multi-node designing of cloud system is commonly used to provide protection against single node failure. Redundancy nodes designing reduces the probability of a cloud system failure. If the failure of any one element of the cloud system could cause the overall system to cease functioning, it is known as a "single point of failure." Fault tree can give two available evaluation: qualitative and quantitative. The farmer assessments give a qualitative ranking of each component in regard to its contribution to failure[8], while quantitative assessments determine if reliability requirements of the component are fulfilled or not. It is suggested to find out which component or module mostly contributes in system unreliability in order to provide a solution in improving its reliability. So, reliability is a nonfunctional characteristic and a term that is focused in the ability of a product to fulfill a certain function[9].

In this paper, multi-node SDDC design is proposed to solve single point of failure. For simply, we evaluate reliability through calculating the MTTF values. After discussing and analyzing the results of the calculation we can conclude that the reliability of multi-node SDDC is better than single node. 


\section{Reliability needs of Multi-Node SDDC system and Fault Tree analysis}

The basic idea behind the multi-node SDDC is that two or more all kinds of resource nodes are used together to provide redundant control. One node is normally active, processing system service requests, while the other is dormant, waiting for the other to fail. A graphical model of parallel and sequential fault combinations is the fault tree model which denotes the occurrence of a predefined undesired event. Its usage is based on failure of all kinds components which are hardware failures, human mistakes or other events. The fault tree describes the logical interrelationships of these basic events that lead to the undesired event, which is the TOP event of the tree. The TOP event (named based on its position on the tree) corresponds to a whole system failure. So, the tree is composed of all the components that cause this event. Fault trees include a set of entities, known as "gates" that allow or prevent logic flow in the tree. Gates show the relations between the necessary entities, which cause another event higher in the tree to happen. This event is considered to be the gate output, while the other events are gate inputs. The symbol of the gate specifies the relation type between the necessary inputs for a certain output[10].

\section{Reliability analysis of multi-node SDDC system using fault tree}

The SDDC users are unable to make an right decision about choosing the right means to face fault tolerance depending on the types of failures that they face. These failures can be network failures, server failures or others. The fault tree can trace the reasons due to node failure. Today more and more researchers turn their eyes on the reliability. In this section, we discuss the idea of reliability. We concentrate on the analysis and comparison of single node and multi-node SDDC system.In order to correctly establish the reliable model, we need to look at the reliability of the role of various factors. we use MTTF (Mean Time to Failure) which is the average time of normal operation before system failure to scale the reliability of the system.

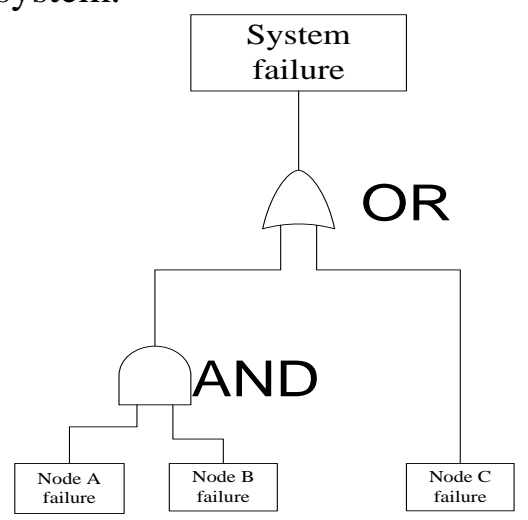

Fig. 1 The fault tree model of multi-node cloud data center

$$
\begin{aligned}
R_{\mathrm{MNsystem}} & =p(\overline{(\text { system failure })} \\
= & p(\overline{\text { A failure } \bullet B \text { failure }+C \text { failure })} \\
= & p(\overline{\text { A failure }}+\overline{B \text { failure }}) p(\overline{C \text { failure }}) \\
= & \left(R_{A}+R_{B}-R_{A} \cdot R_{B}\right) R_{C} \quad\left(R_{A}=R_{B}=R\right) \\
= & \left(2 R-R^{2}\right) R_{C} \\
= & \left(2 e^{-\lambda_{c} t}-e^{-2 \lambda_{c} t}\right) e^{-\lambda_{r} t} \\
R_{\text {SNsystem }} & =p(\overline{\text { system failure }}) \\
= & p(\overline{\text { A failure }+C \text { failure })} \\
= & p(\overline{\text { A failure }}) \cdot p(\overline{C \text { failure }}) \\
= & R_{A} R_{C} \\
= & e^{-\lambda_{c} t} e^{-\lambda_{r} t}
\end{aligned}
$$

We will use fault tree to build reliability model for multi-node data center. This paper only consider AND-gates and OR-gates. Figure 1 shows the fault tree model of multi-node SDDC. 
Assuming using $R_{d}$ presents reliability of node $d$ from 0 to $t$ time, the fault rate of node is $\lambda_{\mathrm{c}}$, the fault rate of other component is $\lambda \mathrm{r}$, and the failure probability of all nodes are same. We can calculate MTTF of multi node and single node respectively, as follows:

Where, MN represents multi node and SN represents single node. A and B represents two nodes respectively. C represents other component. Using (1) and (2), MTTF can be calculated as follows:

$$
\begin{aligned}
\operatorname{MTTF}_{\mathrm{MN}} & =\int_{0}^{\infty} p(\text { running to } t) d t \\
& =\int_{0}^{\infty} R_{\mathrm{MNsystem}} d t=\int_{0}^{\infty}\left(2 e^{-\lambda_{c} t}-e^{-2 \lambda_{c} t}\right) \cdot e^{-\lambda_{r} t} d t \\
& =\frac{2}{\lambda_{c}+\lambda_{r}}-\frac{1}{2 \lambda_{c}+\lambda_{r}} \\
\operatorname{MTTF}_{\mathrm{SN}} & =\int_{0}^{\infty} p(\text { running to } t) d t \\
& =\int_{0}^{\infty} R_{S N \text { system }} d t=\int_{0}^{\infty} e^{-\lambda_{c} t} \cdot e^{-\lambda_{r} t} d t \\
& =\frac{1}{\lambda_{c}+\lambda_{r}}
\end{aligned}
$$

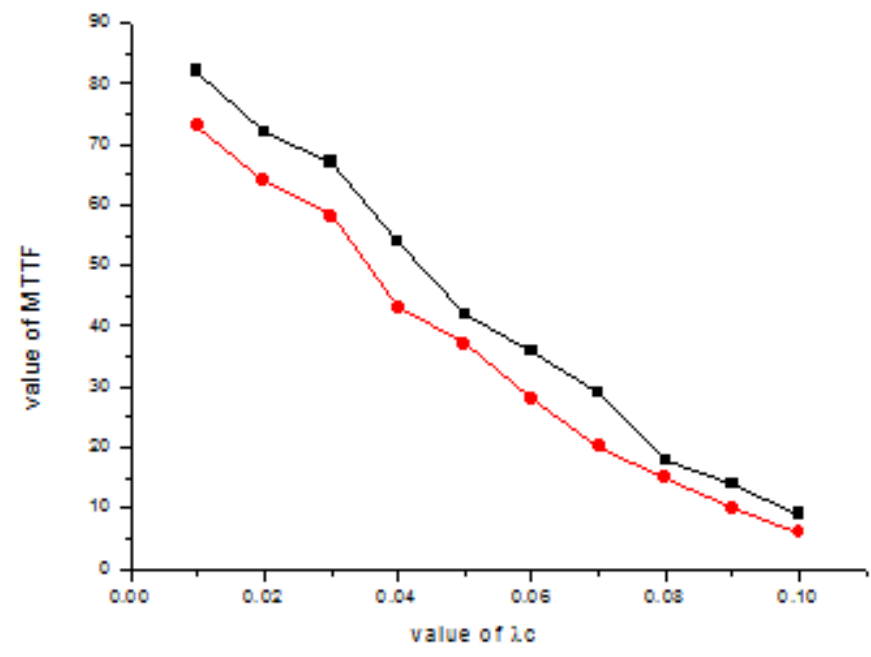

Fig.2 MTTR based on the different values of $\lambda_{c}$

We assume that the failure rate of node $\lambda_{\mathrm{r}}=0.01$. According to the values of different $\lambda_{\mathrm{c}}$, we can compare reliability of multiple and single node.In the same way, assuming $\lambda_{\mathbf{c}}=0.01$, we input different values of $\lambda \mathrm{r}$, which can make us know component failure to the performance. It is showed in figure 2 and 3. From figure 2, we can conclude clearly that the reliability of multi nodes is better than single node. Figure 3 shows that MTTF is affected with different values of $\lambda \mathrm{r}$. Especially, when $\lambda_{\mathrm{r}}$ reach 0.03 , the difference of reliability between dual multi nodes and single node is not obvious. It's showed that when other component failure rate is higher than node failure rate, the effect to reliability of system of multi nodes is same as single node.

\section{Conclusion}

Designing a cloud data center to meet dependability goals in a multi-application environment is difficult. The performance and reliability all are the goals of near-optimal multi nodes designs. Multi nodes is designed for improving reliability and availability of cloud subsystem. This paper use fault tree to scale reliability of multi nodes. In the subsequent study, we will focus on security and elastic deployment, which also are the key technologies of SDDC cloud system. 


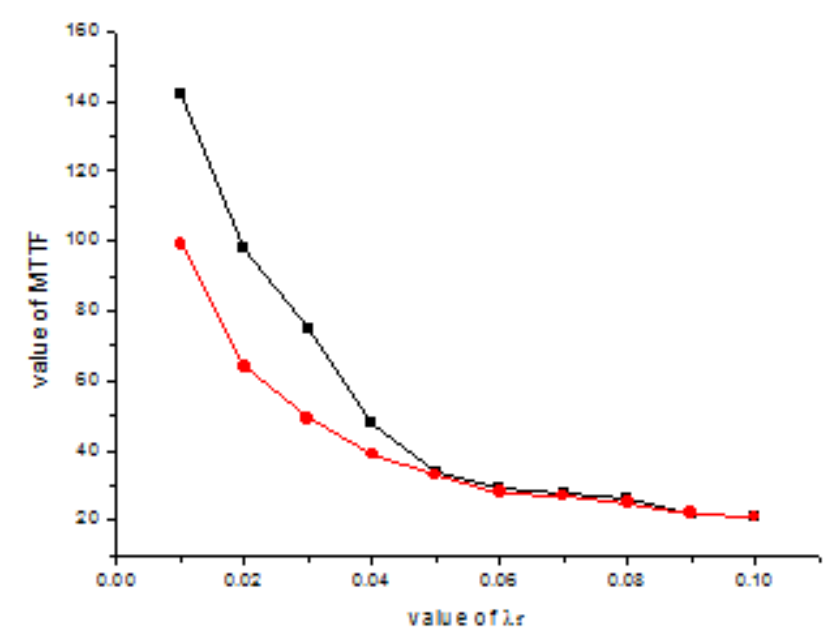

Fig.3 MTTR based on the different values of $\lambda_{r}$

\section{References}

[1] R.Raghavendra, P. Ranganathan, V. Talwar, Z. Wang, X.Zhu. No Power Struggles: Coordinated multi-level power management for the data center. In Thirteenth International Conference on Architectural Support for Programming Languages and Operating Systems (ASPLOS ’08), Mar.2008.

[2] J. Koomey.Growth in data center electricity use 2005 to 2010.in Analytics Press, 2011.

[3] N. Ram Ganga Charan, S. Tirupati Rao, Dr .P.V.S Srinivas, "Deploying an Application on the Cloud", The International Journal of Advanced Computer Science and Applications, (IJACSA), Vol. 2, No. 5, 2011

[4] Tchana, A.; Broto, L.; Hagimont, D.; , "Approaches to cloud computing fault tolerance,"Computer, Information and Telecommunication Systems (CITS), 2012 International Conference on , vol., no., pp.16,1416 May 2012

[5] Farzad Sabahi.Cloud Computing Reliability, Availability and Serviceability (RAS): Issues and Challenges. International Journal on Advances in ICT for Emerging Regions.2011, 04 (02):1223

[8] M. Armbrust, A. Fox, R. Griffith, A. D. Joseph, R. Katz, A. Konwinski, G. Lee, D. Patterson, A. Rabkin, I. Stoica, and M. Zaharia, “A view of cloud computing,” Commun ACM, vol. 53, no. 4, pp. 50-58, Apr. 2010.

[9] M. Alhamad, T. Dillon, and E. Chang, "Service Level Agreement for Distributed Services: A Review," in Dependable, Autonomic and Secure Computing, IEEE International Symposium on, Los Alamitos, CA, USA, 2011, vol. 0, pp. 1051-1054.

[10]United States Nuclear Regulatory Commission, "Fault Tree Handbook", NUREG - 0492, 1993

[11]Elinda Kajo Meçe, Eneida Driza. An Approach to Evaluate the Reliability of Web Applications in Cloud Computing Using Dynamic Fault Tree. BCI'13 September 19-21, Thessaloniki, Greece. 